\title{
THE SUBJECTIVE WELL-BEING OF THE HOMELESS, AND LESSONS FOR HAPPINESS
}

(Accepted 9 June 2005)

\begin{abstract}
The current study assessed the subjective well-being of a broad spectrum of homeless people. One-hundred-and-eighty-six homeless people from the streets of Calcutta (India), California, and a tent camp in Portland (Oregon) were interviewed, and responded to measures of subjective well-being. They answered questions about life satisfaction, satisfaction with various life domains, and their experience of positive and negative emotions. The mean rating of life satisfaction was slightly negative for both American samples but positive for the pavement dwellers in Calcutta. Satisfaction with self-related domains was positive, whereas satisfaction with material related domains was generally negative. Satisfaction with social domains appears to be the area of largest variation among the groups. We discuss the importance of social factors and basic material needs as they relate to overall subjective well-being of the homeless.
\end{abstract}

KEY WORDS: homeless, quality of life, subjective well-being, well-being

\section{SUBJECTIVE WELL-BEING OF THE HOMELESS}

Poverty is one of the most pressing social concerns in the world today. In 1999, according to the United Nations Human Development Report (2002), nearly half the people in sub-Saharan Africa and more than a third of those in South East Asia lived on less than a dollar a day. Of the poor, the most visible are the homeless. Whether they are gangs of street youth or panhandling drifters, no society is unaffected by the social problem of homelessness. There is little agreement about the possible causes and solutions to this social ill. Although past research has focused on psychopathology, incidence of trauma, and the demography of the homeless, little attention has been paid in the psychological literature to the overall quality of life of the homeless. Instead, the bulk of the research literature has been confined to clinical aspects of homelessness or to the effects of 
homelessness on children (e.g., Aptekar, 1994). It is often assumed that life on the street is fraught with difficulty, but little information has been collected to suggest which specific life domains might be the least problematic, and in which areas, if any, the homeless might actually be flourishing. Unfortunately, overlooking the possible resources and strengths of the homeless limits our ability to create effective interventions.

The existing literature on homelessness strongly suggests that there are many problems associated with life without a home. Studies have shown that homelessness is associated with problem behaviors in children (Edleman and Mihaly, 1989; DiBiase and Waddell, 1995), strained family relationships (Vostanis et al., 1996; Nyamathi et al., 1999), higher exposure to trauma (Hien and Bukszpan, 1999; Buhrich et al., 2000), increased anger and depression (Marshall et al., 1996), and the negative psychological impact of social stigma (Lankenau, 1999). Because of the methodological difficulties related to studying homelessness, it is unclear whether factors such as depression and alcohol abuse are causes or effects of homelessness. While prior history of mental illness is undoubtedly responsible for homelessness in at least some cases, there is evidence to suggest that the experience of homelessness causes or exacerbates many psychological problems. In a study by Shlay (1994), for example, homeless people were found to report greater emotional well-being and fewer behavioral problems in their children after positive changes in their economic and social status. The need for research on personal resources and successes on the street becomes more pressing to the extent that people can overcome the psychological ills that accompany homelessness.

Studies on the harmful effects of homelessness are consistent with a larger body of literature examining the relation between income and subjective well-being. In large national surveys, for example, income has been shown to be moderately correlated with life satisfaction, especially at the lower economic levels and in the poorest countries (Diener et al., 1999; Diener and Lucas, 2000; Diener and BiswasDiener, 2002). Higher income has been shown to be related to increased longevity (Wilkenson, 1996), better health (Salovy et al., 2000), and greater life satisfaction (Diener et al., 1985; Diener and Oishi, 2000). Scholars appear to agree that although correlations between income and subjective well-being are often modest, there appears to be a curvilinear relationship in which money has the 
greatest impact on psychological health at the lowest economic levels (Inglehart and Klingemann, 2000).

Two theories are often advanced to explain these findings: basic needs and adaptation. In the former theory, it is assumed that basic physical needs such as food, water, and shelter, must be satisfied before a person can attain higher order psychological fulfillment (Maslow, 1954). By this reasoning, homeless individuals, who face impediments to fulfilling basic needs, should exhibit lower levels of subjective well-being. As homeless people gain better access to food and shelter there ought to be a corresponding increase in psychological health. The theory of adaptation is also helpful in understanding the relation between income and subjective well-being. Research on adaptation suggests that diminished responsiveness to repeated stimuli allows people to adjust to life circumstances, including adverse circumstances (Silver, 1982; Loewenstein and Frederick, 1999). But while people can often adapt relatively well to discrete instances of trauma, there are conditions to which it is more difficult to adapt. Stroebe et al. (1996), for instance, found that widows show higher average levels of depression than their non-bereaved counterparts, even 2 years after the death of their spouse. A review of income and national happiness data by Diener and Diener (1995) showed lower levels of SWB in poor nations, suggesting that extreme poverty is a condition difficult to adapt to, even in the long run.

The current study is, in part, an exploration of the limits of adaptation. We hope to begin to answer the questions of which life domains, including physical needs such as food and social needs such as friendship, are necessary for long-term subjective well-being. By examining the emotional quality of life of groups living in extreme deprivation, we have the opportunity to assess both potential adaptation and the fulfillment of basic needs as they influence subjective well-being. Only by turning research attention on tragic but common cases in which people do not have enough, or have barely enough, basic needs fulfillment will we be able to articulate how "basic" and how "needed" are food, shelter, and social relationships.

Our prior research with homeless people (Biswas-Diener and Diener, 2001) suggests that social factors may be of more fundamental importance than Maslow (1954) believed. According to the findings of our earlier study, social networks play a key role in overall sub- 
jective well-being. This is consistent with recent research by Diener and Seligman (2002) suggesting that good social relationships are necessary for high subjective well-being. Similarly, some theorists argue that value judgments could be as important to emotional wellbeing as basic needs (Brown, 1996). A sense of personal failure in the eyes of society, for example, can be psychologically and socially debilitating. A study of homeless people who later found housing showed that improved quality of life depended on "what kind of housing was obtained," with people transitioning to independent living situation making much larger gains in satisfaction than those transitioning to dependent living situations (Wolf et al., 2001). To the extent that social and self-actualization needs are fundamentally important to subjective well-being, an examination of specific life domains might help shed light on which aspects of the homeless experience are most beneficial, and which are most detrimental, to the emotional quality of life of people on the streets.

One of the primary difficulties in understanding the specific experience of homelessness is the fact that homeless people are a heterogeneous group (Brown, 1996). In the United States there is wide variation in age, gender, and ethnicity of homeless people, as well as causes of homelessness that include unemployment, disability, mental illness, domestic violence, and poor social networks (Hagen, 1987). In other countries there are distinct categories of homeless people ranging from "street children" (Aptekar, 1994) to sex workers (Biswas-Diener and Diener, 2001). Likely, there are substantial differences in the experience of these disparate groups, as well as their collective resources for coping with homelessness. A complete understanding of the homeless experience is beyond the scope of any single study, but would be greatly aided by large surveys of homeless populations. In an early example of one such study we attempted to capture the similarities and differences in the experience of the poorest citizens of Calcutta, including slum dwellers, pavement dwellers, and sex workers (Biswas-Diener and Diener, 2001). While certain elements of life were consistent among the three groups, such as concerns related to income, there were also marked differences in experience, such as the perception of social stigma specifically related to prostitution.

The current study attempts to examine a broad spectrum of the homeless experience by sampling three diverse groups: pavement 
dwellers in Calcutta (India), homeless people (pavement and shelter dwellers) in Fresno, California (USA), and homeless people residing in a self-governing tent camp in Portland, Oregon (USA). Although these three groups do not represent the entire range of the homeless experience, they are sufficiently diverse to enable a better understanding of which aspects of homelessness are universal and which may be unique to specific demographic groups.

In addition, the current study is intended to provide important information useful for policy decisions and applications of interventions related to the homeless. Past research demonstrates that the public is concerned about the problem of homelessness (Morgan et al., 1997) but may have lost the compassion necessary to motivate helping behaviors (Link et al., 1995). In survey data collected in Fresno among a sample of people patronizing a filling station $(N=29)$, we found that these members of the "normal" population were widely misinformed about both the causes of homelessness, and the ways in which homeless people gain income (Biswas-Diener and Diener, 2000). Sixteen of our respondents identified drug abuse and 12 identified laziness as the major cause of homelessness, while physical disability was mentioned by only one person (21 of our 78 homeless respondents received government disability payments). Similarly, 21 of 29 pilot respondents mentioned "begging" as a source of income for the homeless (only four of our 78 homeless respondents reported panhandling, while 36 earned money from day labor). To the extent that helping behaviors are guided by sympathy, empathy, and a realistic understanding of the issues faced by homeless people, an examination of homeless quality of life is an important addition to the research literature. Our study was aimed both at furthering our understanding of the homeless experience and at the more general issues related to how conditions affect well-being.

\section{METHODS}

In the current study, we sampled from diverse groups of homeless people. One hundred and eighty six homeless people were interviewed using a variety of subjective well-being measures (described in detail below) including those assessing general life satisfaction, satisfaction with specific domains of life, and positive and negative affect. The 
measures were presented in written form for literate research participants, and in interview format for illiterate participants. There were scattered missing data for respondents, resulting in slightly lower N's for several of the analyses.

Research participants came from one of three groups: pavement dwellers from Calcutta (India), homeless people from Fresno (California), and people residing at "Dignity Village," a self governing tent camp for homeless people in Portland, Oregon (USA). Interviews with the respondents from both of the American samples were conducted by the first author at or near the dwelling places of the research participants. The Indian research participants were interviewed at or near their dwellings by a trained, experienced research assistant fluent in Bengali, Hindi, and English.

Respondents in Fresno were recruited by the first author at Poverello House, an agency providing free meals and other services for poor and homeless people. Female participants in Fresno were recruited either from Poverello House or the nearby Holy Cross Center for Women, a social service agency providing aid to poor and homeless women and their children. Interviews were conducted privately, and participants were offered minor compensation for their participation. Five people refused to participate in the study, but three refusals were due to an inability to speak English fluently. Participants from Dignity Village were recruited on-site by the first author, and offered a small amount of money for participation in the study. Literate participants provided written answers to surveys, while illiterate participants answered the same questions in interview format. Only two people refused to participate at Dignity Village.

In India respondents were recruited by word-of-mouth by a trained research assistant at the Dum Dum railway platform and from a footpath near the Marwari Hospital (both locations in Calcutta). Twelve people refused to participate, citing either concerns about what would be done with the research results (many pavement dwellers live in illegal settlements), or an inability to understand the research because of illiteracy. Interviews were conducted in relative privacy, away from other homeless people.

Each homeless group is described in detail below:

1. Pavement dwellers in Calcutta. The term "pavement dweller" refers to individuals and families in Calcutta who reside on sidewalks, 
railway platforms, parks, and street medians rather than in permanent or semi-permanent shelters. By some estimates there are at least 100,000 homeless children and 200,000 refugee pavement dwellers in Calcutta (Thomas, 1999). Pavement dwellers typically possess some form of bedding and a tarpaulin or mosquito net for protection against the elements. Those pavement dwellers residing together with their family members often possess pots and other simple cooking utensils, and must forage for scrap wood or cardboard to provide fuel for a cooking fire. Those female pavement dwellers who are employed usually occupy positions related to domestic service (e.g., cooking and cleaning), while males are frequently rickshaw pullers or market employees. Pavement dwellers are vulnerable to police harassment and inclement weather. The current sample $(N=67)$ includes 25 men and 42 women who range in age from 15 to 70 .

2. Homeless men and women in Fresno, California. The homeless population of Fresno, California is a relatively heterogeneous group. The men and women who obtain meals at Poverello House are married, divorced, and widowed; young and old; Spanish and English speaking; and of many ethnicities. Further, some of them receive public welfare benefits, while others find work as day laborers, and a few find steady employment driving taxis, engaging in prostitution, selling drugs, or working at traveling carnivals. Likewise, the Fresno homeless have a variety of sleeping arrangements, ranging from homeless shelters and temporary rented rooms to tent camps and abandoned cars and buildings. The relatively mild weather in California allows the homeless to sleep outside more comfortably than their peers in our other two samples. The Fresno homeless have certain elements in common as well: the vast majority reported a history of sexual or physical abuse, a history of drug use, a history of arrests and/or incarceration, and complain frequently of police harassment. The incidence of violent crime is relatively high in Fresno, and the homeless are at particular risk. The research participants frequently complained of a lack of trust of their peers and the need for constant vigilance. The homeless people in Fresno have relatively few possessions - usually only clothes, a coat, and a bedroll - but they have relatively good access to services including free dental care, hair cuts, religious ministry, food, and blankets. The current sample $(N=78)$ includes 62 men and 16 women who range in age from 21 to 62 . Care was taken to ensure that women and men, and 
people of all ethnicities and ages had the opportunity to participate in the study.

3. Dignity Village, Portland, Oregon. Dignity Village (DV) is a self-governing cooperative tent community in Portland, Oregon. It has legal non-profit status and was founded by the homeless as a transitional living arrangement for homeless adults. DV is unusual in the United States in that it is was founded by homeless people, for homeless people, and is populated and governed by homeless people. Despite general prohibitions against long-term camping in public spaces the City of Portland has allowed DV to locate in a parking lot near the airport and adjacent to a state prison. According to the Dignity Village charter, residents are obligated to donate time to DV community activities including security, fund raising, sanitation, and outreach. Residents of DV live in tents or wooden structures made from discarded scrap wood. These structures are elevated off the ground by wooden palettes to protect them the frequent flooding that accompanies the heavy winter rains. Most residents of Dignity Village own bedding, clothing, books, candles, simple cookware, and a few own pets, musical instruments, and bicycles. There are also community-wide resources available to residents of Dignity Village: a basketball hoop, an organic garden, a common area with woodburning stove, and shower and bathroom facilities. In addition, DV has internet access (run from locally generated wind-power), and the residents have made all community facilities wheelchair accessible to accommodate disabled residents. Most DV residents are not employed full time, and report histories of drug abuse and failed relationships. The charter of Dignity Village allows for only 60 full time residents. The current sample $(N=41)$ includes 29 men and 12 women who range in age from 22 to 59 . The sample, according to unpublished statistics kept by the DV governing council, is approximately representative of Dignity Village in terms of age, sex, and race.

\section{Measures}

By using multiple measures we were able to capture both cognitive (satisfaction judgments) and affective (emotions and moods) aspects of subjective well-being. Members of all three samples answered questions related to demographics, life satisfaction, positive 
and negative affect, and domain satisfaction. In addition, research participants in Calcutta and at Dignity Village answered questions relating to history of homelessness, drug use, medical history, family history, education, and current living conditions. All questions were presented in English in the United States and in Bengali or Hindi in India. The translator was an experienced psychological interviewer who had been trained specifically for this interview.

The respondents were approached where they lived or received meals and asked to participate in the study. They were told that their participation was voluntary, and that, in some cases they would receive minor compensation for their participation. All respondents were informed about confidentiality and instructed not to use a family name during the interview. At Dignity Village, where literacy was higher than either of the other two groups, informed consent forms were used. Verbal consent was obtained in Fresno and Calcutta. Participants were told they were free not to answer any given question and that they could ask questions about the research following the interview.

The interview began with the collection of basic demographic information, a brief biography, and questions related to current living conditions, arrest history, medical history, and drug history. Next, the respondents were presented with the Satisfaction With Life Scale (SWLS), a 5-item measure of general life satisfaction found to have good psychometric properties (Pavot and Diener, 1993). A 1-7 Likert scale was used for each of the items, but segmented rectangles were used for illiterate respondents. The rectangles were tested for comprehension prior to the interview and have been used in other research with non-literate populations (Biswas-Diener et al., 2004, Submitted).

Following the SWLS, the participants were asked to respond to questions about the frequency of their experience of positive and negative emotions (affection, joy, sadness, anger, guilt, worry, and pride) during the previous month. They answered using a 1 (never) to 7 (always) Likert scale. Past research has shown cultural variation in which emotions are considered core pleasant or unpleasant feelings (e.g., Kim-Prieto et al., 2004, submitted). Due to these differences in the interpretation of the emotion "pride," in particular, we chose not to include it with either positive or negative emotions in our analyses. 
The participants were then asked to rate their satisfaction with 14 life domains (material resources, friendship, morality, intelligence, food, romantic relationship, family, physical appearance, self, income, housing, social life, privacy, and health). The domains were presented in the same order to all respondents, who were asked to rate their satisfaction with each on a 1 (totally dissatisfied) to 7 (totally satisfied) scale. Identical measures of domain satisfaction have been used in past research with homeless groups (e.g., Biswas-Diener \& Diener, 2001).

\section{RESULTS}

\section{Demographic Variables}

Demographic information for each group is presented in Table I. As can be seen, men outnumbered women in the American samples, a fact true of homeless people in the United States in general. It is possible that men are underrepresented in the Indian sample because the interviewer was a woman and same sex contacts were more comfortable for both the interviewer and interviewees. Data compiled from the Fresno Rescue Mission, a shelter agency in Fresno, shows that our sample approximates their service population for gender and age, but that Hispanic people are under-represented in our sample (Hispanic people constitute approximately $41 \%$ of all

TABLE I

Demographic variables

\begin{tabular}{lllll}
\hline & Calcutta & Dignity village & Fresno & Total \\
\hline $\mathrm{N}$ & 67 & 41 & 78 & 186 \\
Men & 25 & 29 & 62 & 116 \\
Women & 42 & 12 & 16 & 70 \\
Age & 37.4 & 40.3 & 44 & 40.7 \\
& $(12.0)$ & $(10.2)$ & $(9.0)$ & $(10.8)$ \\
\% Caucasian & & $83 \%$ & $31 \%$ & \\
\% Married & $72 \%$ & $41 \%$ & & \\
Average Monthly & 24 dollars & 270 dollars* & 358 dollars* & \\
Income & & & & \\
\hline
\end{tabular}

*Includes government benefits such as "food stamps." 
shelter stays; Fresno Rescue Mission web-site, 2001). Reasons for Hispanic under-representation could include English language comprehension difficulties, or temporary work that interfered with participation in the research. The Dignity Village sample was representative of Dignity Village as a whole on all key demographic variables.

\section{Life Satisfaction}

The means and standard deviations for key satisfaction variables are presented in Table II. The means appear in the left hand column and the standard deviations appear in parentheses to the right. We conducted an analysis of the SWLS' internal reliability with each of

\section{TABLE II}

Satisfaction of the homeless

\begin{tabular}{llll}
\hline Group: & Calcutta & Dignity village & Fresno \\
\hline Life Satisfaction & $22.20(8.68)^{\mathrm{A}}$ & $17.27(7.36)^{\mathrm{B}}$ & $14.12(7.61)^{\mathrm{B}}$ \\
$\begin{array}{l}\text { (5-35; 20 is neutral) } \\
\text { Domain Satisfaction }\end{array}$ & & & \\
(score range1-7) & & & \\
Material Domains & & & \\
1. Material Resources & 3.46 & 3.84 & 3.42 \\
2. Food & 5.37 & 4.41 & 4.99 \\
3. Income & $3.75^{\mathrm{A}}$ & $2.41^{\mathrm{B}}$ & $2.68^{\mathrm{B}}$ \\
4. Housing & 2.38 & 3.28 & 3.17 \\
5. Health & $3.33^{\mathrm{A}}$ & $4.63^{\mathrm{B}}$ & $4.62^{\mathrm{B}}$ \\
Social Domains & & & \\
6. Social & $5.08^{\mathrm{A}}$ & $4.34^{\mathrm{B}}$ & $4.04^{\mathrm{B}}$ \\
7. Romantic Relations & 4.97 & 4.19 & 4.04 \\
8. Friends & 4.22 & 5.00 & 4.07 \\
9. Privacy & 3.00 & 3.09 & 3.22 \\
10. Family & $5.93^{\mathrm{A}}$ & $4.31^{\mathrm{B}}$ & $4.36^{\mathrm{B}}$ \\
$\quad$ Self-related Domains & & & \\
11. Self & $5.77^{\mathrm{A}}$ & $4.66^{\mathrm{B}}$ & $4.73^{\mathrm{B}}$ \\
12. Morality & 4.97 & 5.41 & 4.57 \\
13. Physical Appearance & 4.40 & 4.34 & 4.36 \\
14. Intelligence & 5.85 & 5.00 & 5.38 \\
\hline
\end{tabular}

Note: Scores in the same row with different superscript letters differ from each other by $p<0.05$ or less. 
the three groups. For the Calcutta sample the Cronbach's alpha was 0.78. For Dignity Village the Cronbach's alpha was 0.78 . The Satisfaction with Life Scale showed the best reliability with the Fresno homeless population with an overall alpha of 0.84 . The substantial alphas in all three samples suggested that the items formed a coherent whole.

An ANOVA was performed to determine whether or not the three groups differed from one another on general Life Satisfaction, and the significant overall $F(2,179)=18.16, p<0.001$ was followed up by a Bonferroni post hoc multiple comparison to determine which groups differed from one another. The Calcutta pavement dwellers scored the highest on life satisfaction $(M=22.2)$, Dignity Villagers in the middle $(M=17.27)$, and the Fresno homeless lowest $(M=14.12)$. Because the neutral point of the SWLS is 20.0, only the pavement dwellers in Calcutta scored in the positive range, and both the Fresno homeless and the Dignity Villagers scored significantly lower than the Calcutta group $(p<0.001$ and $p<0.01$, respectively).

The mean scores for Domain Satisfaction are also shown in Table II. All three samples scored in the positive range on the majority of domains except, as expected, on material satisfaction, income, housing, privacy, and health (Calcutta only). The only domain related to material satisfaction in which the three samples scored positively was food. A MANOVA was performed across the domains to ascertain whether the groups differed significantly from one another. The overall significant MANOVA (Pillai's Trace Criterion, $F(28,292)=3.02, p<0.001)$ was followed up by a Bonferroni post hoc comparison of the three means for each domain, and the significant differences between groups are shown in Table II. The Calcutta sample was found to differ significantly from the other two groups on satisfaction with income, social life, family, health and self. Of these, it was only on health satisfaction that the Calcutta respondents scored significantly lower.

The means for the frequency of experience of positive and negative affect are shown in Table III. The overall MANOVA $[F(14$, $288)=1.55, p<0.10]$ was followed by post hoc comparisons of the groups for each emotion; although the overall MANOVA was only of marginal significance. As can be seen in Table III, only a few significant differences were found between the three sample groups. It 
TABLE III

Positive and negative affect of the homeless

\begin{tabular}{llll}
\hline & Calcutta & DV & Fresno \\
\hline 1. Affection & $3.65^{\mathrm{A}}$ & $4.62^{\mathrm{A}}$ & $4.68^{\mathrm{B}}$ \\
2. Joy & 4.08 & 3.95 & 4.22 \\
3. Anger & 4.18 & 3.72 & 3.94 \\
4. Sadness & $4.48^{\mathrm{A}}$ & $3.54^{\mathrm{B}}$ & $4.06^{\mathrm{A}}$ \\
5. Guilt & 3.44 & 2.92 & 3.44 \\
6. Worry & 4.32 & 4.33 & 4.48 \\
7. Pride & 3.95 & 3.90 & 4.56 \\
\hline
\end{tabular}

Scores range from 1 (never) to 7 (always).Scores in the same row with a different superscript letter differ by $p<0.05$ or less.

is noteworthy that all three samples reported unusually high levels of negative affect. In fact, the levels of NA were higher than PA in Calcutta, and almost equal to PA in the U.S. samples. In all three samples in the current study the frequency of worry exceeded that of joy. These results differ markedly from most samples, where the difference between pleasant and unpleasant emotion scores is strongly positive (Diener and Diener, 1996).

\section{Variables Associated with Basic Needs}

Because basic physical needs such as adequate shelter have long been assumed to be a prerequisite for psychological health, special attention to variables associated with basic needs is warranted. As can be seen in Table II the three samples were generally dissatisfied with their material domains of life. The high level of reported food satisfaction is a notable exception. In Calcutta a single basic needs variable in Calcutta - crowding - predicted general life satisfaction, but not in the expected direction. The total number of people sharing pavement space with the research participant correlated significantly and positively with general life satisfaction $(r=0.37$, $p<0.05$ ), with the more people sharing space reporting higher life satisfaction! It is possible that this finding is caused by the social benefits of living with friends and family members. Housing appeared to be an important part of overall subjective well-being in the American samples: Not only was satisfaction with ones' own 
housing a strong predictor of general life satisfaction, but with satisfaction with many other life domains as well. At Dignity Village housing satisfaction correlated with general LS $(r=0.40$, $p<0.05)$, as well as with self satisfaction $(r=0.45, p<0.05)$. In Fresno, housing satisfaction correlated significantly (at the $p<0.001$ level $)$ with general life satisfaction $(r=0.57)$, material satisfaction $(r=0.41)$, food satisfaction $(r=0.46)$, and income satisfaction $(r=0.54)$.

In Calcutta income satisfaction appeared to be extremely important to overall subjective well-being. Income satisfaction correlated significantly (at the $p<0.001$ level) with food satisfaction $(r=0.50)$, health satisfaction $(r=0.47)$, and social satisfaction $(r=0.45)$.

\section{DISCUSSION}

Common sense and common images of homelessness lead us to believe that "life on the street" is extremely undesirable, with both physical and psychological downsides. The results of the current study largely support this conclusion. In both our American samples respondents reported below neutral levels of life satisfaction while all three homeless samples reported high negative affect. Across the three groups the respondents' reported low levels of satisfaction with material resources such as income and housing, where housing referred to sleeping accommodations ranging from tents to makeshift shelters to community shelters. We also found strong correlations between housing satisfaction and general life satisfaction. It is interesting that the three samples in the current study, chosen for their differences, reported similar trends in material satisfaction. This suggests that there are certain experiences common to extreme poverty - such as health problems - that transcend differences in culture. The relatively low satisfaction and high negative affect scores in the current study are consistent with a larger research literature showing a relation between income and subjective well-being (e.g., Diener \& Suh, 1999; Argyle, 2001). Although expected, these results provide additional support for the pressing need to address the problem of homelessness. 
Aside from these intuitive findings there are also some unexpected, and encouraging, results from the current research. For example, all three samples reported surprisingly high satisfaction with domains related to the self, including morality, intelligence, and physical appearance. In fact, scores for all self-related domains were substantially above the neutral point for all three samples. This suggests that despite material and emotional hardships, homeless people tend to like themselves. This is consistent with the literature on the positive aspects of cognition, such as the finding that people tend to believe they are better than average on most dimensions (e.g., Heady and Wearing, 1988). If, in fact, homeless people view themselves in a positive light, as our research suggests, this finding points to a potential area of strength that may be useful in designing policies and interventions targeting homelessness. This finding also indicates directions for future research, suggesting that self-appraisal may be an aspect of psychology largely invulnerable to the negative effects of poverty.

Another interesting finding from the current study is the high level of reported satisfaction with food by members of all three of samples in the current study. Although the three groups were, on average, dissatisfied with all domains related to material resources, including income and housing, all three reported above-neutral satisfaction with their food, despite large differences in quality, quantity, and food security. This is consistent with past research, in which groups of homeless people have reported high satisfaction with food (e.g., Marshal et al., 1996). Why would food satisfaction be one of the most satisfying domains for the homeless people in our study? One possibility is that the expectations of these groups are so low that any amount or quality of food is appreciated. Another possible explanation is that these groups are actually hungry, so that they truly enjoy the food they eat. In the case of the U.S. samples, social service agencies and government issued food coupons might provide ample food that is beyond the level of resources obtained in other areas. Further research needs to be conducted to better understand the unusually high food satisfaction scores of the homeless.

Perhaps the most counter-intuitive finding is the relatively high subjective well-being of our sample in India. Despite poorer access to food, clean water, medical care, opportunities for employment, and adequate shelter than their counterparts in the United States, the 
pavement dwellers in Calcutta reported higher levels of life satisfaction. Not only was the general life satisfaction among the Calcutta sample higher than that of the two American samples, but it was in the positive range! This is consistent with our past research, in which impoverished groups in Calcutta reported surprisingly high life satisfaction, given their environmental conditions (Biswas-Diener and Diener, 2001). What could account for this unexpected positive quality of life? The answer may lie in specific life domains.

Despite similar trends among the 3 samples in self satisfaction, the Calcutta sample reported significantly greater satisfaction in this area. And, although we found a common pattern of dissatisfaction with material domains, the Calcutta sample reported significantly higher satisfaction with income. Finally, while people in both American samples reported satisfaction with social domains that was near or below the neutral point, the Calcutta sample expressed significantly greater satisfaction with both their overall social lives and their families, well above the neutral point in both instances. In addition, sharing living quarters with many other people in Calcutta was correlated with increased life satisfaction. This suggests that the relations among pavement dwellers in Calcutta are of a higher quality than among those in the United States, where respondents commonly complained of mistrusting others. Further support for this can be found in the fact that the Calcutta respondents reported higher levels of affection than did members of the other two homeless groups. This is consistent with our past results from research with homeless people in Calcutta in which we suggested good social relations may help protect against the harmful psychological effects of poverty (BiswasDiener and Diener, 2001).

There are many possible explanations for the relatively high social satisfaction of the Calcutta sample, and we discuss 2 of them here: (1) The state of West Bengal, in which Calcutta is located, has a communist government that may be associated with widespread sympathy for the poor, and therefore fewer feelings of persecution and marginalization by the poor. In addition, because of the high overall rate of poverty in India in general, and Calcutta in particular, there might be less social stigma attached to homelessness, whereas similar social standing in America is often seen as the result of personal failure. This is supported by the finding that the Calcutta sample reported significantly higher levels of satisfaction with "self" than the 
other two groups. (2) Homelessness in Calcutta is often the result of macroeconomic conditions, such as high competition for jobs, rather than with personal pathology such as alcoholism, mental illness, or physical disability. Because of this, homeless people in Calcutta tend to stay together as family units, whereas their counterparts in the Untied States frequently report being estranged from spouses, children, and friends. Support for this explanation of higher life satisfaction can be found in the fact that the Calcutta sample reported significantly higher satisfaction with "family" than the U.S. samples.

More research needs to be conducted before we can determine the causes of the unusually high social satisfaction and self satisfaction of the Calcutta homeless sample. Regardless of the exact cause, though, it seems likely that social satisfaction is an important component to overall subjective well-being, and may be especially so for homeless individuals. This is consistent with the work of Sheldon et al. (2001) in which they suggest that "relatedness" rivals physical needs as a fundamental human need, as well as that of Diener and Seligman (2002), in which they found that good relationships are necessary for happiness. The results of the current research suggest that social needs merit renewed attention, and suggest that they may be as important as physical needs.

Our findings run counter to Maslow's (1954) classic basic needs theory, in which he suggested that physical and material needs must be met before social and psychological health can occur. Although Maslow's theory is widely accepted, little is understood about the specific thresholds for "meeting needs." For instance, do people need to eat one meal a day before they are able to develop healthy social relationships? What if they only eat every-other day? Our research findings suggest that physical, social, and psychological needs might interact in a more "horizontal" fashion to create overall psychological well-being, and that a success in one can counterbalance a failure in another. Indeed, we do not argue here that material needs are unimportant, or less important than social needs, but that the two may deserve an equal place in the hierarchy of psychological health. While some physical needs such as a healthy body and adequate housing are important for subjective well-being, the results of the current study suggest the possibility that we are less psychologically vulnerable to material deprivation than previously thought, at least in comparison to social needs. 
It is also possible that adaptation plays a role in subjective wellbeing as it relates to basic needs. Results from previous research suggests that people have an extraordinary ability to adapt to certain physical events such as a handicap (Silver, 1982), but that they adapt less well to social ills such as the death of a spouse (Stroebe et al., 1996). It could be that a lack of basic material needs takes a psychological toll, but that people adapt rather well to it as compared to a lack of good social relationships. It is noteworthy that with extremely low income the Calcutta sample shows higher income satisfaction than the other two samples, indicating the importance of one's standards to satisfaction judgments. Further research needs to be conducted to examine the role of adaptation in meeting basic needs as well research focusing on the relation of physical, social, and existential needs to one another.

In the end, our research reveals certain common characteristics of homeless people in diverse settings: they are dissatisfied with their material quality of life, especially their housing, income, and health. Similarly, they are highly satisfied with themselves: their morality, physical appearance, and intelligence. Unfortunately, liking oneself does not seem sufficient to counterbalance the psychological harm caused by material deprivation. Good social relationships, on the other hand, are not only important to subjective well-being (Diener and Seligman, 2002) but may to some extent avert the psychological costs of material deprivation.

\section{ACKNOWLEDGEMENTS}

The authors would like to thank in Fresno: Robert Levine, Mike McGarvyn and Poverello House, The Holy Cross Center for Women, and Ari. At Dignity Village: Jack Tafari, Gaye, Tim, Ibrahim, and all the research participants. In Calcutta: Avirupa Bhaduri

\section{REFERENCES}

Aptekar, L.: 1994, 'Street children in the developing world: A review of their condition', Cross-Cultural Research: The Journal of Comparative Social Sciences 28, pp. $195-224$. 
Argyle M.: 2001, The Psychology of Happiness, 2nd edition (New York, Routledge).

Biswas-Diener, R. and E. Diener: 2000 [A survey of attitudes about homelessness]. Unpublished raw data.

Biswas-Diener, R. and E. Diener: 2001, 'Making the best of a bad situation: Satisfaction in the slums of Calcutta', Social Indicators Research 55, pp. 329-352.

Brown, I.: 1996, The quality of life of marginal citizens, in R. Renwick, I. Brown and M. Nagler (eds.), Quality of Life in Health Promotion and Rehabilitation (Sage Publications, Thousand Oaks), pp. 204-216.

Buhrich, N., T. Hodder and M. Teesson: 2000, 'Lifetime prevalence of trauma among homeless people in Sydney', Australian \& New Zealand Journal of Psychiatry 34 , pp. 963-966.

Christensen, R.C. and G.D. Grace: 1999, 'The prevalence of low literacy in an indigent psychiatric population', Psychiatric Services 50, pp. 262-263.

DiBiase, R. and S. Waddell: 1995, 'Some effects of homelessness on the psychological functioning of preschoolers', Journal of Abnormal Child Psychology 23, pp. 783792.

Diener, E. and R. Biswas-Diener: 2002, 'Will money increase subjective well-being?: A review and guide to needed literature', Social Indicators Research 57, pp. 119169.

Diener, E. and C. Diener: 1995, 'The wealth of nations, revisited: Income and quality of life', Social Indicators Research 36, pp. 275-286.

Diener, E. and R.E. Lucas: 2000, 'Explaining differences in societal levels of happiness: Relative standards, need fulfillment, culture, and evaluation theory', Journal of Happiness Studies 1, pp. 41-78.

Diener, E., J. Horowitz and R. Emmons: 1985, 'Happiness of the very wealthy', Journal of Personality Assessment 49, pp. 71-75.

Diener, E. and E.M. Suh: 1999, National differences in subjective well-being, in D. Kahneman, E. Diener and N. Schwarz (eds.), Well-Being: Foundations of Hedonic Psychology (Russell Sage Foundation, New York), pp. 434-450.

Diener, E., E.M. Suh, R.E. Lucas and H.E. Smith: 1999, 'Subjective well-being: Three decades of progress', Psychological Bulletin 125, pp. 276-302.

Diener, E. and S. Oishi: 2000, Money and Happiness: Income and subjective wellbeing across nations, in E. Diener and E.M. Suh (eds.), Culture and Subjective Well-Being (MIT press, Cambridge, MA), pp. 185-218.

Diener, E. and M.E.P. Seligman: 2002, 'Very happy people', Psychological Science 13 , pp. 80-83.

Edleman, M.W. and L. Mihaly: 1989, 'Homeless families and the housing crisis in the Untied States', Children \& Youth Services Review 11, pp. 91-108.

Frederick, S. and G. Loewenstein: 1999, Hedonic adaptation: From the bright side to the dark side, in D. Kahneman, E. Diener and N. Schwarz (eds.), Well-Being: Foundations of Hedonic Psychology (Russell Sage Foundation, New York), pp. 302-329.

Fresno Rescue Mission: 2001, Fresno Rescue Mission Statistics 1999 [On-line]. Available: http://www.fresnorescuemission.org/.

Hagen, J.L.: 1987, 'The heterogeneity of homelessness', Social Casework 68, pp. 451457.

Heady, B. and A. Wearing: 1988, 'The sense of relative superiority - Central to wellbeing', Social Indicators Reserach 20, pp. 497-516. 
Hien, D. and C. Bukszpan: 1999, 'Interpersonal violence in "normal" low-income groups', Woman \& Health 29, pp. 1-16.

Inglehart, R. and H-D Klingemann: 2000, Genes, culture, democracy, and. Happiness, in E. Diener and E. M. Suh (eds.), Culture and Subjective Well-Being (MIT Press, Cambridge, Massechusettes), pp. 165-183.

Lankenau, S.E.: 1999, 'Stronger than dirt: Public humiliation and status enhancement among panhandlers', Journal of Contemporary Ethnography 28, pp. 288318.

Link, B.G., S. Schwartz, R. Moore and J. Phelan: 1995, 'Public knowledge, attitudes, and beliefs about homeless people: Evidence for compassion fatigue', American Journal of Community Psychology 23, pp. 533-555.

Marshall, G.N., M.A. Burnam, P. Koegel, G. Sullivan and B. Benjamin: 1996, 'Objective life circumstances and life satisfaction: Results from the Course of Homelessness Study', Journal of health \& Behavior 37, pp. 44-58.

Maslow, A.H.: 1954, Motivation and Personality (Harper \& Row, New York).

Morgan, M.M., H.W. Goddard and S.N. Givens: 1997, 'Factors that influence willingness to help the homeless', Journal of Social Distress \& the Homeless 6, pp. $45-56$.

Nyamathi, A., S. Wenzel, C. Keenan, B. Leake and L. Gelberg: 1999, 'Associations between homeless women's intimate relationships and their health and well-being', Research in Nursing \& Health 22, pp. 486-495.

Pavot, W. and E. Diener: 1993, 'Review of the Satisfaction with Life Scale', Personality Assessment 5, pp. 164-172.

Salovey, P., A.J. Rothman, J.B. Detweiler and W.T. Steward: 2000, 'Emotional states and physical health', American Psychologist 55, pp. 110-121.

Sheldon, K.M., A.J. Elliot, Y. Kim and T. Kasser: 2001, 'What is so satisfying about satisfying events? Testing 10 candidate psychological needs', Journal of Personality and Social Psychology 80, pp. 325-339.

Shlay, A.: 1994, 'Running on empty: Monitoring the lives and circumstances of formerly homeless families with children', Journal of Social Distress \& the Homeless 3, pp. 135-162.

Silver, R.L.: 1982, Coping with an Undesirable Life Event: A Study Of Early Reactions to Physical Disability (Doctoral Dissertation- Northwestern University, Evanston, Illinois).

Stroebe, W., M. Stroebe, G. Abakoumkin and H. Schut: 1996, 'The role of loneliness and social support in adjustment to loss: A test of attachment versus stress theory', Journal of Personality and Social Psychology 70, pp. 1241-1249.

Thomas, F.C.: 1999, Calcutta: The Human Face of Poverty (Penguin Books, Calcutta, India).

United Nations (United Nations Development Program, 2002). Human Development Report: Deepening Democracy in a Fragmented World. New York: United Nations.

Vostanis, P., S. Cumella, J. Briscoe and F. Oyebode: 1996, 'A survey of psychosocial characteristics if homeless families', European Journal of Psychiatry 10, pp. 108117.

Wilkenson, R.G.: 1996, Unhealthy Societies: The Affliction of Inequality (Routledge, London).

Wolf, J., A. Burnam, P. Koegel, G. Sullivan and S. Morton: 2001, 'Changes in subjective quality of life among homeless adults who obtain housing: A prospec- 
tive examination', Social Psychiatry and Psychiatric Epidemiology 36, pp. 391398.

University of Oregon

Robert Biswas-Diener

E-mail:jayajedi@comcast.net

Department of Psychology

Ed Diener

University of Illinois and The Gallup Organization 603 E. Daniel Street

Champaign, IL 61820

USA

E-mail: ediener@cyrus.psych.uiuc.edu 